\title{
Metamaterial-based perfect absorbers for efficiently enhancing near field radiative heat transfer
}

\author{
Nan Zhou, Xianfan $\mathrm{Xu}^{*}$ \\ School of Mechanical Engineering and Birck Nanotechnology Center, Purdue University, \\ West Lafayette, IN, USA 47907
}

\begin{abstract}
:
The fascinating capability of manipulating light using metamaterials (MMs) has inspired significant amount of studies of using MMs for energy related applications. In this work we investigate MM-based perfect absorbers for enhancing near field radiative heat transfer, which is described by the fluctuation dissipation theorem. MM structures designed at two wavelengths are analyzed, corresponding to two working temperatures. Both electric and magnetic surface polaritons are found to contribute to heat transfer, while natural materials support only electric polaritons. The near-perfect absorption is demonstrated to be related to the modification of effective optical properties, which is important for enhancing radiative heat transfer efficiently. By comparing different designs, the bandwidth of the heat flux spectrum is found to increase with the absorption bandwidth, which is originated from the spatial field distributions. This study will contribute to the understanding of surface polaritons in near field radiative heat transfer and facilitate the optimization of MMs for near field heat transfer applications.
\end{abstract}

\section{Keywords:}

radiative heat transfer, metamaterials, near field, surface polaritons

\section{Introduction:}

The demonstration of negative refractive index materials [1-3] has generated significant amount of interests in researches of metamaterials (MMs) [4-7]. The fascinating light manipulation capability of MMs has many potential applications related

*Corresponding author. Tel: 1-765-494-5639, Fax: 1-765-494-0539, Email address: xxu@ecn.purdue.edu. 
energy utilization. For example, MMs can be designed for radiation selective surfaces tailoring the solar absorption and emission of light at a particular wavelength, i.e., selective absorbers or emitters [8-13]. This holds the potential in the field of thermal photovotaics, where the conversion efficiency could be significantly enhanced if the wavelength is matched to the bandgap of photovoltaic materials $[14,15]$.

Nano-structured surfaces or meta-surfaces can also be designed to enhance near field radiative heat transfer (NRHT). Polder and Van Hove [16] found a heat flux increase between two parallel plates, separated by a distance smaller than $\lambda_{T}$ due to the contribution from evanescent waves, where $\lambda_{T}$ is the peak wavelength of the thermal radiation spectrum given by the Wien's displacement law: $\lambda_{T} T \approx 2898 \mu \mathrm{m} \cdot \mathrm{K}$. Recent work has shown that it is possible to enhance spectral heat flux by several orders of magnitude through resonantly excited surface phonon polaritons (SPhPs) in polar materials such as $\mathrm{SiC}$ and glass [17-19]. The intrinsic optical properties of polar materials limit the SPhPs to around10 $\mu \mathrm{m}$, corresponding to an operating temperature close to the room temperature. Other materials investigated in the infrared range include doped $\mathrm{Si}$ [20, 21] and graphene $[22,23]$, which enhance NRHT through exciting surface plasmon polaritons (SPP).

For noble metals that also support SPP, the resonance typically lies in the visible or UV wavelengths, which is significantly above the thermal frequency range. This corresponds to an operating temperature higher than 2,000 K which could melt the metal, thus limits the direct application of metallic materials in NRHT. However, by applying nanostructures, the response can be strongly manipulated through the interaction between incident electromagnetic waves and the properly designed structures. The incident light can be coupled to not only electric surface polaritons, but also magnetic polaritons [11, 13]. This method provides flexibility in tailoring the optical properties in a wide frequency range and thus provides a potential to effectively enhance NRHT.

Analysis of the NRHT usually employs fluctuational electrodynamics [24], which has been extended to MMs with arbitrary permittivity $\varepsilon$ and permeability $\mu$ by Joulain et al. [25]. In classical electromagnetics, the Drude-Lorentz model is applied to $\varepsilon\left(=\varepsilon^{\prime}+i \varepsilon^{\prime \prime}\right)$ and $\mu\left(=\mu^{\prime}+i \mu^{\prime \prime}\right)$, derived from the oscillation of charges or fictitious magnetic charges. For a dilute metal design with extremely low plasma frequency [26], an analytically 
expression was derived for the effective permittivity $\varepsilon_{\text {eff, }}$ which still obeys the DrudeLorentz model. Similarly, the average response of split ring resonator (SRR) MMs was also analytically characterized [27]. More generally, analytical solutions are not available for the collective optical response of inhomogeneous structures. Instead, the effective medium theory (EMT) is widely applied to homogenize the structure with the aid of the geometric filling ratio [28-30] or the S-parameters from numerical simulation [15, 31, 32]. It is accepted that EMT is generally applicable if the incident wavelength is much larger than size of the unit cell, and the distance to the structure surface is not smaller than the size of the unit cell [29-31]. Many researchers have developed alternative approaches for evaluating the MM-based NRHT with improved accuracy at small plate separations. A scattering approach [33] based on rigorous coupled-wave analysis (RCWA) has been applied to compute the NRHT between metallic [34] and dielectric [35] gratings. Wen [36] conducted direct simulations for NRHT between two parallel plates using Wiener chaos expansion, which has no explicit constraints on the geometry and relies on finding eigenmodes of the thermal fluctuating current. Liu and Shen [37] extended this method to hyperbolic MM consist of metallic wire array. Other methods for solving NRHT problems in arbitrary configurations include direct finite-difference time-domain (FDTD) computations [38, 39], boundary-element method (BEM) [40], and thermal discrete dipole approximation (T-DDA) [41].

In this study we focus on MMs that have a top metallic antenna array and a bottom metal ground layer, separated by a dielectric spacer. This type of MMs has been demonstrated both numerically and experimentally to provide near perfect absorption and functions as a blackbody at the desired wavelength $[11,15]$. We extend the study of these MMs to the field of near field radiative heat transfer between parallel plates, through enhancing the strength of surface polaritons and thus enhancing the NRHT. We also investigate the effect of temperature and compare different MM designs.

\section{Methods and Geometry:}

The parallel plates geometry under study is illustrated in Fig. 1, where the medium 3 between two plates is typically vacuum with $\varepsilon_{3}=1$. By applying the fluctuation dissipation theorem, the net radiative heat flux is given as [42]: 


$$
\begin{aligned}
P\left(T_{1}, T_{2}\right) & =\pi \int_{0}^{\infty} d \omega\left[I_{\omega}^{0}\left(T_{1}\right)-I_{\omega}^{0}\left(T_{2}\right)\right] \\
& \times \sum_{\alpha=s, p}[\underbrace{\int_{0}^{\omega / c} \frac{K d K}{(\omega / c)^{2}} \frac{\left(1-\left|r_{31}^{\alpha}\right|^{2}\right)\left(1-\left|r_{32}^{\alpha}\right|^{2}\right)}{\left|1-r_{31}^{\alpha} r_{32}^{\alpha} e^{2 i \gamma_{3} d}\right|^{2}}}_{\text {Propagating }}+\underbrace{\int_{\omega / c}^{\infty} \frac{K d K}{(\omega / c)^{2}} \frac{4 \operatorname{Im}\left(r_{31}^{\alpha}\right) \operatorname{Im}\left(r_{32}^{\alpha}\right)}{\left|1-r_{31}^{\alpha} r_{32}^{\alpha} e^{-2 \gamma_{3}^{\prime \prime} d}\right|^{2}} e^{-2 \gamma_{3}^{\prime \prime} d}}_{\text {Evanescent }}],
\end{aligned}
$$

where $K$ is the in-plane wave vector and $I_{\omega}^{0}(T)=\hbar \omega^{3} / 4 \pi^{3} c^{2} /\left(e^{\hbar \omega / k_{B} T}-1\right)$ is the intensity of blackbody radiation, which serves as a $\omega$-domain filter that cuts off modes with $\omega>>k_{B} T / \hbar$ [42]. Both propagating $(K<\omega / c)$ and evanescent $(K>\omega / c)$ waves are accounted in Eq. (1). The denominator involving Fresnel reflection coefficients corresponds to multiple reflections. When the surface plasmon/phonon polaritons are excited, $\operatorname{Im}\left(r^{s}\right)$ is negligible while $\operatorname{Im}\left(r^{p}\right) \approx 2 \operatorname{Im}(\varepsilon) /|\varepsilon+1|^{2}[17]$. As a result, the p-polarized evanescent channel peaks at the same condition as the surface modes that originated from plasmon or phonon polaritons $(\varepsilon=-1)$, significantly enhancing the NRHT. Similar analysis can be applied to the s-polarized channel if the plate has a negative permeability $\mu^{\prime}$, indicating the existence of surface magnetic polaritons. Thus, instead of applying naturally existed bulk materials, we design nanostructures or MMs to manipulate both $\varepsilon$ and $\mu$. The regions with both negative $\varepsilon^{\prime}$ or $\mu^{\prime}$ will considerably enhance the NRHT.

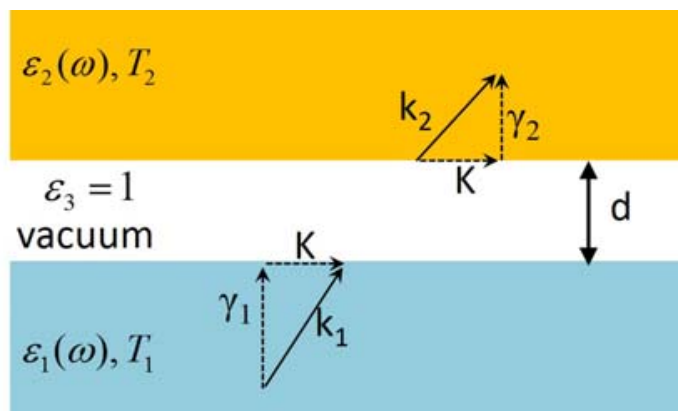

Fig. 1. (single column) Schematic of the nanoscale thermal radiation between two parallel plates. 
According to the analysis in [31], the effective refractive index $\left(n=n^{\prime}+i n^{\prime \prime}\right)$ and impedance $\left(z=z^{\prime}+i z^{\prime \prime}\right)$ are related to the complex reflection coefficient $r$ and transmission coefficient $t$ by

$$
\begin{aligned}
& \cos \left(\mathrm{nk}_{0} D\right)=\frac{1-r^{2}+t^{2}}{2 t} \\
& z= \pm \sqrt{\frac{(1+r)^{2}-t^{2}}{(1-r)^{2}-t^{2}}}
\end{aligned}
$$

Here, $D$ is the total thickness of the structure, and $k_{0}$ is the wave vector in vacuum. The passive medium assumption requires that $z^{\prime}>0$ and $n^{\prime \prime}>0$. We simulated the unit cell of the structure and calculated $r$ and $t$ coefficients using ANSYS HFSS, a commercial frequency-domain finite-element method solver. With $n$ and $z$, the effective optical properties can be readily computed using:

$$
\begin{aligned}
& \varepsilon=n / z \\
& \mu=n \cdot z .
\end{aligned}
$$

MM-based perfect absorbers/emitters are typically made of a top metallic antenna array and a bottom metal ground layer, separated by a dielectric spacer. The unique nearperfect absorption/emission properties take advantages of the electric resonance in the top nano-structure, as well as the magnetic resonance which induces anti-parallel currents in the two metallic layers $[9,11]$. Electric and magnetic resonances can be separately tuned in different elements and simultaneously achieved [8]. Effectively, the structure provides an impedance matching to the free space with $\varepsilon=\mu$, which makes the perfect absorption possible [8, 32]. Various MM patterns have been studies, including cross [10, 15, 43], patch [12, 32, 44], disk [9], and hole [45]. In addition to the strong manipulation of absorption spectrum, MM-based absorbers can be designed to be angular independent or omnidirectional under both TE and TM illuminations, as has been demonstrated experimentally $[12,9,44]$. 
In this work we study two resonant wavelengths: $10 \mu \mathrm{m}$ and $4 \mu \mathrm{m}$. They correspond to working temperatures of about $300 \mathrm{~K}$ and $700 \mathrm{~K}$, respectively. For the wavelength of $10 \mu \mathrm{m}$, we investigate two cross patterns, called cross A and cross B here, to illustrate the relation between far field perfect absorption and near field enhancement (section 3.1). For the wavelength of $4 \mu \mathrm{m}$, we consider several different top metallic patterns as illustrated in Fig. 2. The cross pattern is similar to the fishnet structure, which has been investigated for near field radiation at near - IR wavelengths [46]. The complementary bowtie aperture (CBA) in Fig. 2(d) has been demonstrated in our earlier work to generate enhanced and confined magnetic field around the metal strip [47]. Table 1 summarizes geometry parameters that have been optimized for each design to achieve near-perfect absorption at the resonance. The pattern size $l$ and period $p$ scale with the resonant wavelength. As has been discussed in literature [15, 32, 44, 45, 47], the absorption spectrum is generally most sensitive to $l, p$ and the spacer thickness $t_{s}$. They determine the response of a single pattern, the interaction between adjacent patterns and the coupling between two metallic layers, respectively. Gold is used as the metallic material. For wavelengths $\lambda<2 \mu \mathrm{m}$, its properties were taken from [48]. For long wavelengths, the Drude model $\varepsilon(\omega)=1-\omega_{p}^{2} / \omega^{2}+i \gamma \omega$ is applied with $\omega_{p}$ and $\gamma$ taken from [49]. The spacer material is typically $\mathrm{SiO}_{2}$ or $\mathrm{Al}_{2} \mathrm{O}_{3}$. Here, $\mathrm{SiO}_{2}$ is applied for designs at 4 $\mu \mathrm{m}$ and its optical properties from [48] are used in the simulation. $\mathrm{SiO}_{2}$ is known to have a strong dispersion in the mid-infrared range, thus $\mathrm{Al}_{2} \mathrm{O}_{3}$ is used instead for designs at 10 $\mu \mathrm{m}$ and its frequency-dependent optical constant is taken from [50].
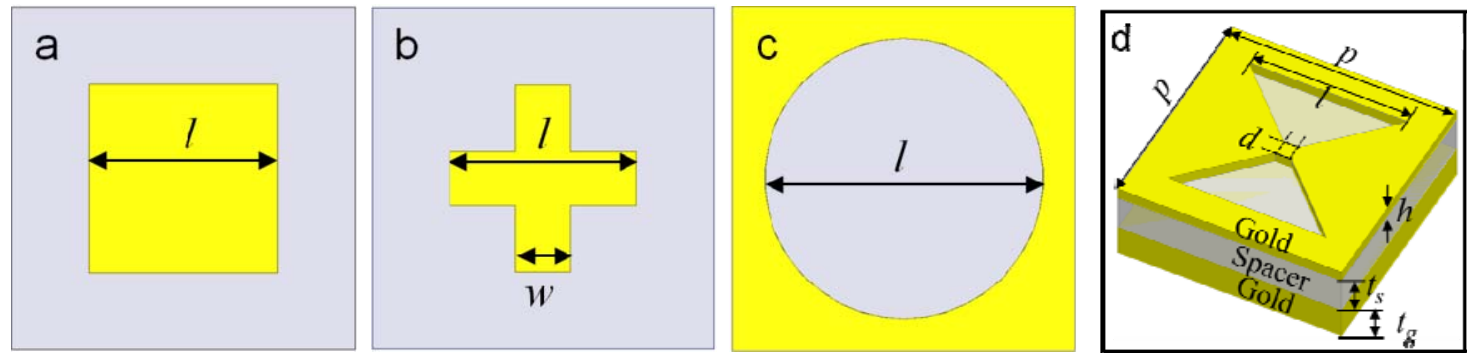

Fig. 2. (2-column) Unit cell of MM patterns made of (a) patch, (b) cross, (c) hole and (d) complementary bowtie aperture. Top view is used for (a-c). The cross-sectional parameters are shown in (d), which is applicable to (a-c). 
Table 1: Geometric parameters for the metamaterial patterns studied in this work.

\begin{tabular}{lllllllll}
\hline Wavelength & Pattern & $\mathrm{t}_{\mathrm{s}}[\mathrm{nm}]$ & $\mathrm{h}[\mathrm{nm}]$ & $\mathrm{t}_{\mathrm{g}}[\mathrm{nm}]$ & $\mathrm{p}[\mu \mathrm{m}]$ & $1[\mu \mathrm{m}]$ & $\mathrm{w}[\mu \mathrm{m}]$ & $\mathrm{d}[\mathrm{nm}]$ \\
\hline \multirow{2}{*}{$10 \mu \mathrm{m}$} & Cross - A & 300 & 100 & 100 & 4.4 & 3.8 & 0.4 & \\
& Cross - B & 100 & & & & & & \\
\hline \multirow{5}{*}{$4 \mu \mathrm{m}$} & Patch & 25 & 40 & 100 & 1.3 & 0.85 & & \\
& Cross & 25 & 40 & 100 & 1.3 & 0.85 & 0.4 & \\
& Hole & 150 & 40 & 100 & 2.7 & 2.6 & & \multirow{2}{*}{0.1} \\
\hline
\end{tabular}

\section{Results and Discussion:}

\subsection{Importance of Perfect Absorption for Near Field Enhancement}

In this section, we investigate two cross patterns A and B that work around the wavelength of $10 \mu \mathrm{m}$, as listed in Table 1 . The importance of strong absorption of MMs on NRHT will be discussed in details by comparing the two designs.

From Table 1 we can see that the only difference between cross A and B is the spacer thickness. Figure 3(a) shows that both designs produce a far field absorption peak at $9.42 \mu \mathrm{m}\left(2 \times 10^{14} \mathrm{rad} / \mathrm{s}\right)$ under normal incidence. But only cross A, with a spacer thickness of $300 \mathrm{~nm}$, achieves near-perfect absorption at resonance. Figures 3(b-d) present the retrieved optical properties of cross A and B, which show effective Lorentz oscillations in the vicinity of $2 \times 10^{14} \mathrm{rad} / \mathrm{s}$. Far away from the oscillation, the absolute value of $\varepsilon$ generally decreases with increasing $\omega$, same as that observed in the Drude model. Thus the effective properties, which are very different from those of bulk or thin film gold, can be well described by the Drude-Lorentz model:

$$
\begin{aligned}
& \varepsilon(\omega)=\varepsilon_{\infty}-\frac{\omega_{\mathrm{D}}^{2}}{\omega^{2}+i \gamma_{\mathrm{D}} \omega}-\frac{\omega_{p, \mathrm{e}}^{2}}{\omega^{2}-\omega_{0, \mathrm{e}}^{2}+i \gamma_{e} \omega} \\
& \mu(\omega)=\mu_{\infty}-\frac{\omega_{p, \mathrm{~m}}^{2}}{\omega^{2}-\omega_{0, \mathrm{~m}}^{2}+i \gamma_{m} \omega} .
\end{aligned}
$$

Here subscript D stands for the Drude term. The corresponding fitting parameters are summarized in Table 2. 
Table 2: Drude-Lorentz fitting parameters for Cross - A and B designs.

\begin{tabular}{lllllll}
\hline & $\varepsilon_{\infty}$ & $\omega_{\mathrm{D}}[\mathrm{rad} / \mathrm{s}]$ & $\gamma_{\mathrm{D}}[\mathrm{rad} / \mathrm{s}]$ & $\omega_{\mathrm{p}, \mathrm{e}}[\mathrm{rad} / \mathrm{s}]$ & $\omega_{0, \mathrm{e}}[\mathrm{rad} / \mathrm{s}]$ & $\gamma_{\mathrm{e}}[\mathrm{rad} / \mathrm{s}]$ \\
\hline Cross - A & 14 & $2 \times 10^{15}$ & $2.8 \times 10^{13}$ & $6.05 \times 10^{14}$ & $2.09 \times 10^{14}$ & $4.25 \times 10^{12}$ \\
Cross - B & 14 & $3.2 \times 10^{15}$ & $2.1 \times 10^{13}$ & $7.25 \times 10^{13}$ & $2.025 \times 10^{14}$ & $6 \times 10^{12}$ \\
\hline & $\mu_{\infty}$ & & & $\omega_{\mathrm{p}, \mathrm{m}}[\mathrm{rad} / \mathrm{s}]$ & $\omega_{0, \mathrm{~m}}[\mathrm{rad} / \mathrm{s}]$ & $\gamma_{\mathrm{m}}[\mathrm{rad} / \mathrm{s}]$ \\
\hline Cross - A & 4.72 & & & $1.43 \times 10^{14}$ & $1.985 \times 10^{14}$ & $5 \times 10^{12}$ \\
Cross - B & 4.16 & & & $0.83 \times 10^{14}$ & $1.978 \times 10^{14}$ & $5.5 \times 10^{12}$ \\
\hline
\end{tabular}

In Figs. 3(b-d), we plot fitting results using parameters in Table 2 together with the directly retrieved properties. For cross A, its near-perfect absorption indicates an effective magnetic Lorentz oscillator that is strong enough to induce negative $\mu^{\prime}$ over a wavelength range. On the other hand, its permittivity is also manipulated from extremely negative values, as observed in bulk gold, to moderate negative $\varepsilon^{\prime}$. This helps enhance the NRHT more efficiently, as indicated by the expression $\operatorname{Im}\left(r^{p}\right) \approx 2 \operatorname{Im}(\varepsilon) /|\varepsilon+1|^{2}$. Compared with cross $\mathrm{A}$, the magnetic oscillator resonance produced by cross $\mathrm{B}$ is much weaker. As a result, $\mu^{\prime}$ stays positive in the simulated spectrum. In addition, $\varepsilon^{\prime}$ is very negative for most of the spectrum. It should be mentioned that $\varepsilon^{\prime}$ will gradually approach -1 on the short wavelength/large frequency side, which will make additional contribution to the pevanescent channel. We ignore this short wavelength resonance because it is outside of the simulation range and it corresponds to a much higher temperature. 

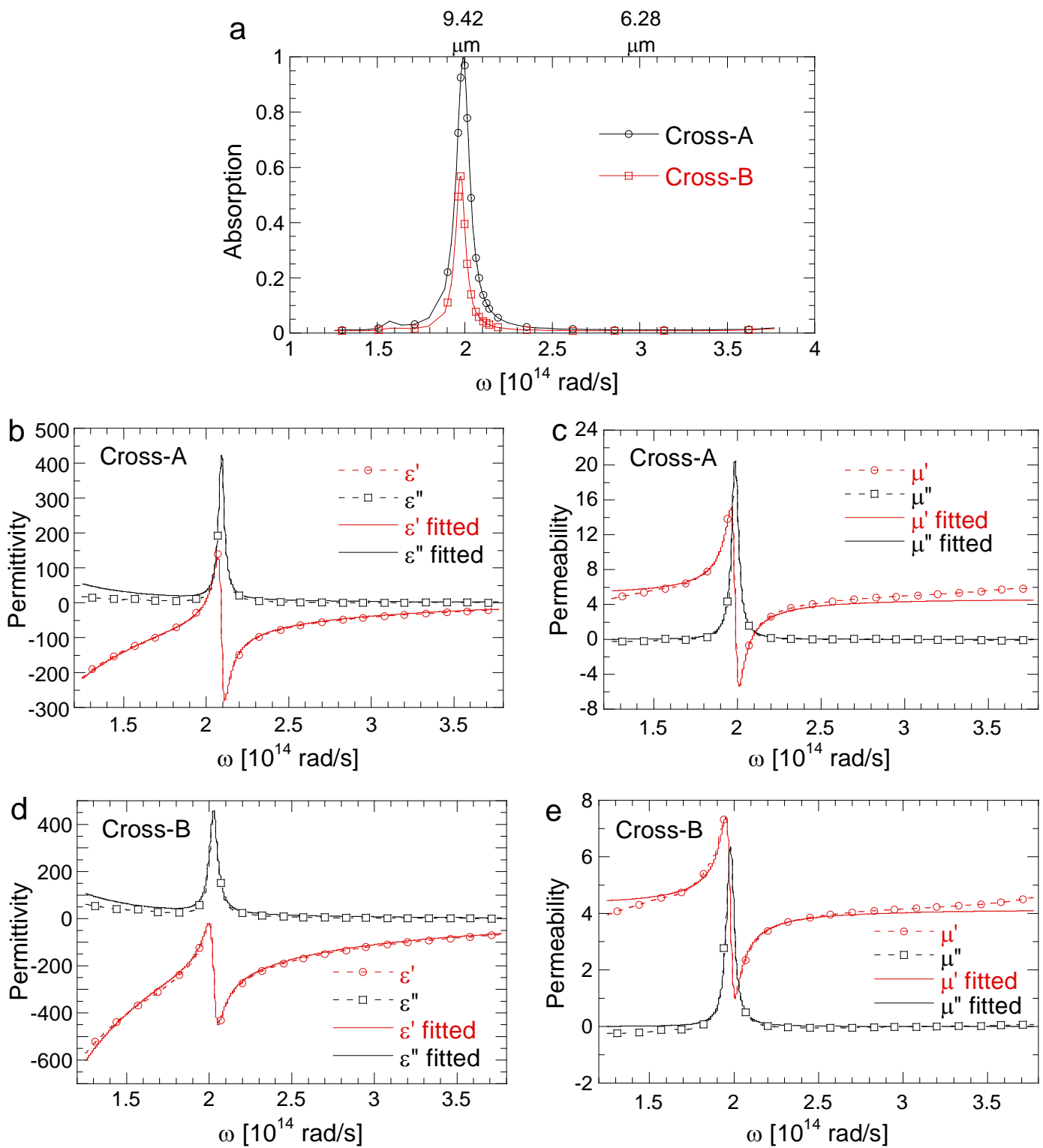

Fig. 3. (2-column) (a) Far field absorption spectra for cross A and B that resonate at $10 \mu \mathrm{m}$. The retrieved and fitted optical properties for $(b, c)$ cross A and $(d, e)$ cross B.

The corresponding NRHT calculations of cross A and B are shown in Fig. 4. The temperatures are fixed at $T_{1}=350 \mathrm{~K}$ and $T_{2}=300 \mathrm{~K}$. Figure $4(\mathrm{a})$ presents the net radiative heat flux as a function of the distance $d$. The heat flux for cross $\mathrm{A}$ is broken down to three channels, the propagating wave, and the s- and p-polarized evanescent waves. The red and green dash lines correspond to s- and p-evanescent modes of cross B, respectively. We can see that both s- and p-evanescent channels of cross A outperform that of B as expected. As the distance increases, the contribution of s-evanescent mode 
decreases rapidly because of the very narrow bandwidth of negative $\mu^{\prime}$ for effectively exciting magnetic polaritons, and the total heat flux is dominated by the $\mathrm{p}$ counterpart and the propagating mode. At $d=4 \mu \mathrm{m}$, close to the period of the designs, the evanescent channels (mainly p-evanescent) contribute about 33\% to the total flux. For the shaded area of $d<4 \mu \mathrm{m}$ in Fig. 4(a), the EMT is not well-suited and the results will likely produce errors for the contribution from evanescent modes. However, we present the corresponding results to explore the effect of optical properties on NRHT, and to obtain a rough idea of the NRHT behavior of the designed structure. It is expected that both electric and magnetic surface polaritons will gradually dominate the heat flux at smaller separations. However, a quantitative analysis is not available with the EMT method.

Figure 4(b) compares the total heat flux of cross A and B with two parallel $\mathrm{Au}$ and $\mathrm{SiC}$ plates. To show a fair comparison, the radiative heat flux is integrated within the same spectral range from $1.2 \times 10^{14} \mathrm{rad} / \mathrm{s}$ to $3.8 \times 10^{14} \mathrm{rad} / \mathrm{s}$ for all cases presented in Fig. 4(b). The surface phonon resonance near $10 \mu \mathrm{m}$ (within the integration range) in $\mathrm{SiC}$ is naturally strong, thus the heat flux between two parallel $\mathrm{SiC}$ plates is larger than that of MMs and bulk gold plates. At $d=4 \mu \mathrm{m}$, the heat flux of cross A is thrice that of B and remains about twice at larger distances. It is also seen that the radiative heat flux between two bulk $\mathrm{Au}$ plates is at least one order of magnitude smaller than that of the properly design MMs. Figure 4(b) indicates that one should still choose SiC over MM-based materials for near-room temperature applications. However, the advantage of using MMbased materials is that their design can be easily modified for application at other wavelength/temperature, or even for multi-/broad-band applications, while the intrinsic surface phonon resonance in $\mathrm{SiC}$ is monochromatic near the $10 \mu \mathrm{m}$ wavelength. 

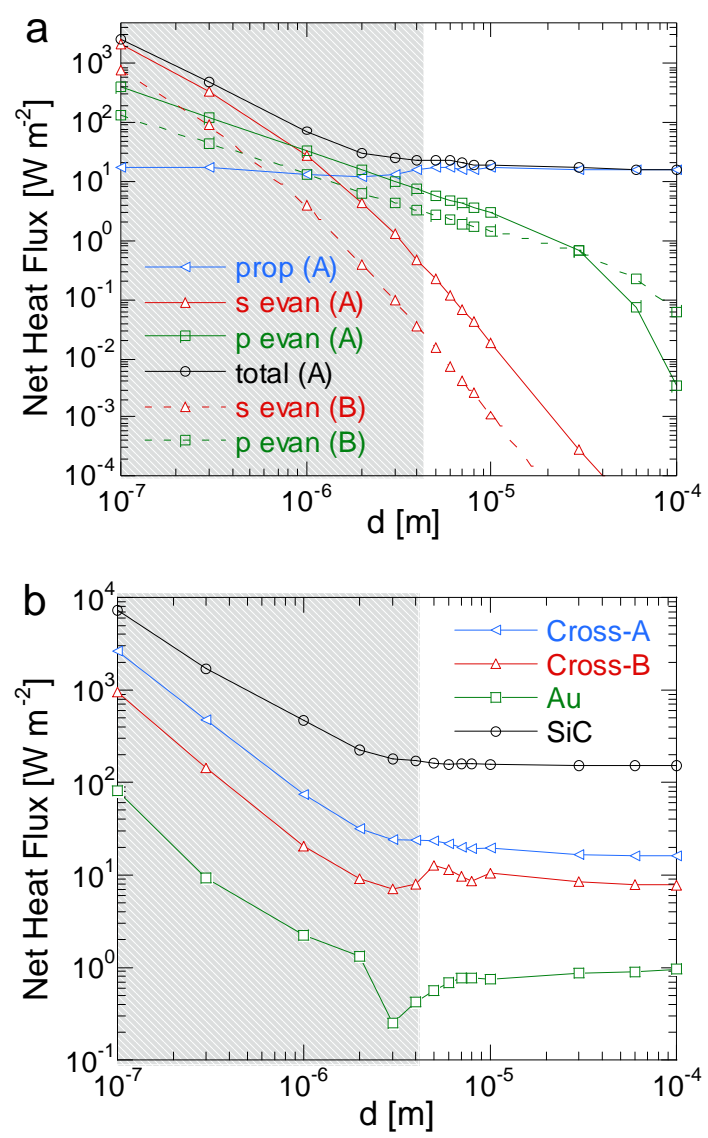

Fig. 4. (single column) (a) The net heat flux of different channels as a function of plate separation for the mid-infrared cross design $\mathrm{A}$. The red and green dash lines correspond to s- and $\mathrm{p}$ evanescent modes of cross B. (b) Compare the total heat flux of cross A and B with that from two parallel $\mathrm{Au}$ and $\mathrm{SiC}$ plates. For all cases in this figure, the temperatures are fixed at $\mathrm{T}_{1}=350 \mathrm{~K}$ and $\mathrm{T}_{2}=300 \mathrm{~K}$.

\subsection{Near-field Enhancement using Different Geometric Designs}

As was mentioned, MM-based perfect absorbers/emitters can have various geometric patterns. In this section, we compare four different patterns shown in Fig. 2 and listed in Table 1. They are all designed around the wavelength of $4 \mu \mathrm{m}$ with a nearperfect absorption at resonance in the far field, as shown in Fig. 5. 


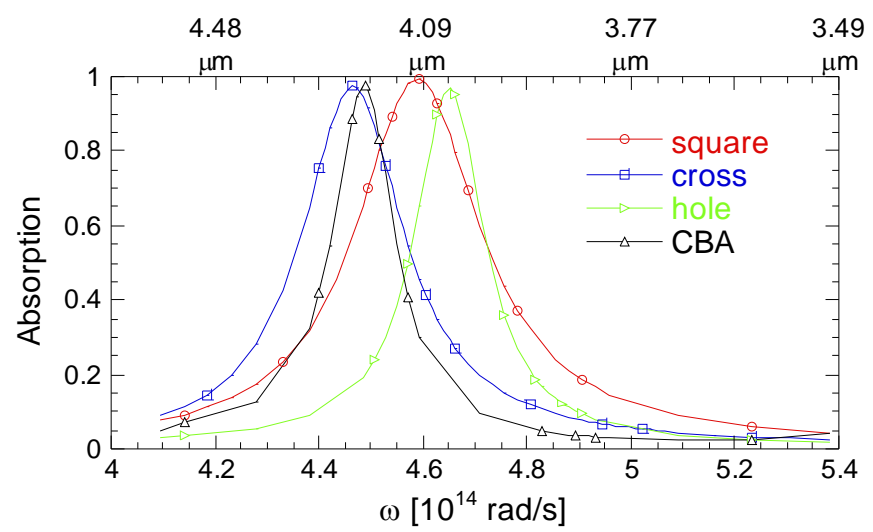

Fig. 5. (single column) Far field absorption spectra for four designs that resonate near $4 \mu \mathrm{m}$.

Figures 6(a) and (b) show the near field heat flux spectra for square patch, cross, hole, and complementary bowtie aperture (CBA) patterns at $d=100 \mathrm{~nm}$ and $2 \mu \mathrm{m}$, respectively. The temperatures are fixed at $\mathrm{T}_{1}=750 \mathrm{~K}$ and $\mathrm{T}_{2}=700 \mathrm{~K}$. The corresponding total heat flux integrated in the spectral range from $4 \times 10^{14} \mathrm{rad} / \mathrm{s}$ to $5.4 \times 10^{14} \mathrm{rad} / \mathrm{s}$ is presented in Fig. 6(c). Although the results at $d=100 \mathrm{~nm}$, which is much smaller than the period, could overestimate the heat flux, they are still used to estimate the geometric effect on NRHT. We see that, firstly, for all designs, two peaks can be observed. The stronger one on the large frequency/short wavelength side corresponds to the s-polarized surface wave, which outperform the p counterpart at small distances. Secondly, we see from Fig. 6(a) that the peak values are slightly different among four designs and the CBA absorber produces the strongest peak (at $4.73 \times 10^{14}$ $\mathrm{rad} / \mathrm{s}$ ). It has been noted that a large loss term of $\varepsilon$ or $\mu$ will deteriorate the contribution of surface modes [30]. Thus, at $d=100 \mathrm{~nm}$, the strong s-evanescent mode from the CBA absorber can be attributed to the relatively small imaginary part in $\mu \approx-1+i 0.4$ (at $4.73 \times 10^{14} \mathrm{rad} / \mathrm{s}$ ). For other designs, $\mu \approx-1+i 0.5$ (at $5.08 \times 10^{14} \mathrm{rad} / \mathrm{s}$ ) for the square, $\mu \approx-$ $1+i 0.84\left(\right.$ at $4.76 \times 10^{14} \mathrm{rad} / \mathrm{s}$ ) for the cross, and $\mu \approx-1+i 1.11$ (at $4.8 \times 10^{14} \mathrm{rad} / \mathrm{s}$ ) for the hole. Thirdly, the full width at half maximum (FWHM) bandwidths $\Delta \omega$ are very different, which were found to be roughly scale with the absorption bandwidth shown in Fig. 5. For square, cross, hole and CBA patterns, the absorption bandwidths $\Delta \omega$ are $2.93 \times 10^{13}$, $2.43 \times 10^{13}, 1.55 \times 10^{13}$, and $1.45 \times 10^{13} \mathrm{rad} / \mathrm{s}$, respectively. The geometric effects on resonance characteristics have also been studied [51], which also reported that the square patch design has a larger absorption bandwidth than cross pattern because of smaller 
effective mutual and kinetic inductances. At a larger distance of $2 \mu \mathrm{m}$, the peaks from evanescent channels significantly decrease, resulting in similar peak values. However, the difference in bandwidth still exists, as shown in Fig. 6(b). The net heat flux at different distances is computed from the spectral response, therefore depending on both the peak value and the bandwidth. Figure 6(c) shows that at $d=2 \mu \mathrm{m}$, the integrated heat flux roughly scales with the bandwidth, while at $d=100 \mathrm{~nm}$, the CBA absorber produces a relatively large heat transfer because of the strong s-evanescent peak shown in Fig. 6(a).
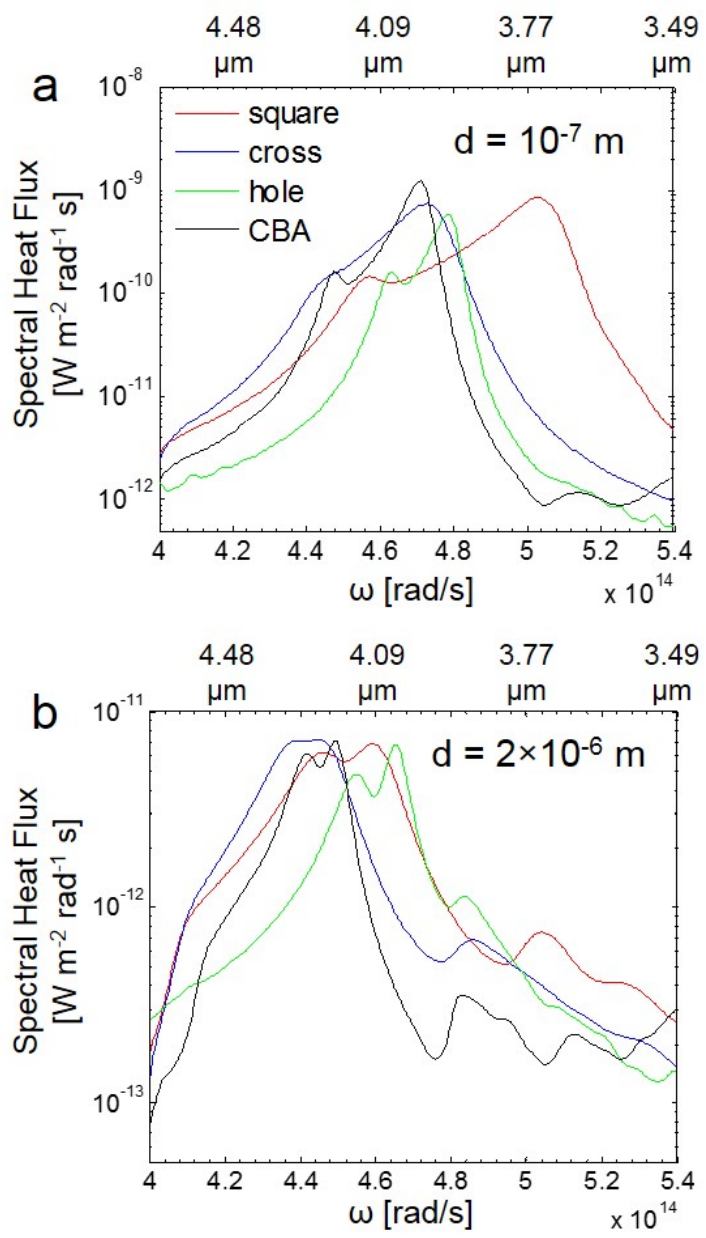

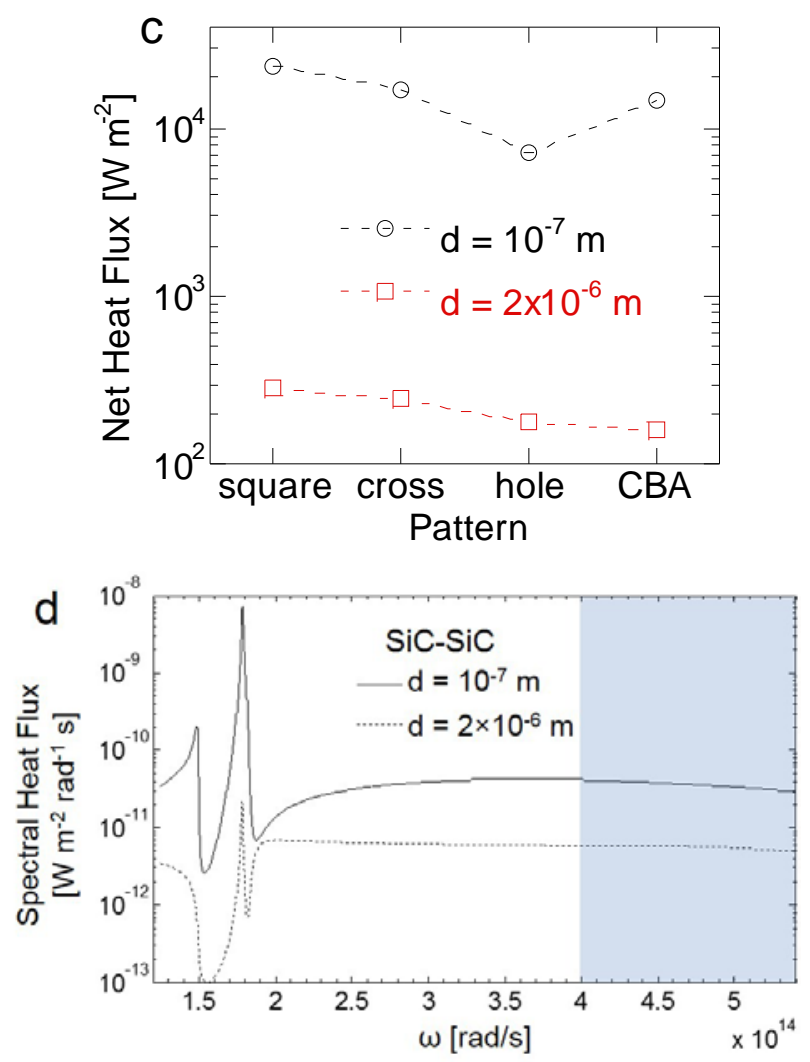

Fig. 6. (single column) The heat flux spectra at (a) $d=100 \mathrm{~nm}$ and (b) $2 \mu \mathrm{m}$ for four designs for 4 $\mu \mathrm{m}$ wavelength. The legend is the same for (a) and (b). (c) The integrated net heat flux at two different distances for four patterns. (d) The heat flux spectra between two SiC plates at two separations. The shaded area indicates the frequency range used for the four MM patterns. For all cases, the temperatures are fixed at $\mathrm{T}_{1}=750 \mathrm{~K}$ and $\mathrm{T}_{2}=700 \mathrm{~K}$.

Lastly, we compare the heat flux of $\mathrm{SiC}$ to the four MM patterns analyzed above, considering temperatures at $T_{1}=750 \mathrm{~K}$ and $T_{2}=700 \mathrm{~K}$. Figure $6(\mathrm{~d})$ shows the heat flux spectra from $1.2 \times 10^{14}$ to $5.4 \times 10^{14} \mathrm{rad} / \mathrm{s}$ between two SiC plates at $d=100 \mathrm{~nm}$ and $2 \mu \mathrm{m}$. The shaded area corresponds to the frequency range used for MM patterns, namely $4 \times 10^{14} \sim 5.4 \times 10^{14} \mathrm{rad} / \mathrm{s}$. There's no surprise that SiC still produces strong surface phonon resonance near $1.8 \times 10^{14} \mathrm{rad} / \mathrm{s}$. At $d=2 \mu \mathrm{m}$, the integrated heat flux is $2.21 \times 10^{3} \mathrm{~W} / \mathrm{m}^{2}$, about 10 times that of MM patterns. At a smaller distance $d=100 \mathrm{~nm}$, the heat flux integrated in the range from $1.2 \times 10^{14}$ to $5.4 \times 10^{14} \mathrm{rad} / \mathrm{s}$ is $2.94 \times 10^{4} \mathrm{~W} / \mathrm{m}^{2}$, which is only slightly higher than the $2.35 \times 10^{4} \mathrm{~W} / \mathrm{m}^{2}$ produced by the patch pattern. For temperatures 
higher than $800 \mathrm{~K}$ or distances less than $70 \mathrm{~nm}$, the MM-based designs provide a higher NRHT than SiC.

\section{Conclusion:}

The MM-based perfect absorbers are applied for enhancing nanoscale radiative heat transfer, using the effective optical properties retrieved from EMT and the fluctuation electrodynamics for parallel plate geometry. The near-perfect absorption by MM, due to the enhanced strength of surface polaritons, is thus related to the efficient enhancement of the near field heat flux. At small plate separations, both electric and magnetic surface modes can contribute to the heat transfer, while the latter does not exist in natural materials. MMs offer great flexibility for tuning resonance in different frequency ranges for enhancing heat flux at different target temperatures. The comparison among different designs reveals that the bandwidth of heat flux roughly scales with the absorption bandwidth, which originates from the spatial field distributions in the unit cell. Therefore, MM absorbers with perfect absorption as well as a broad resonance peak are preferable for NRHT.

\section{Acknowledgements}

The authors gratefully acknowledge the support of the National Science Foundation.

\section{References:}

[1] Pendry JB. Negative refraction makes a perfect lens. Phys Rev Lett 2000;85:3966-9.

[2] Zhang S, Fan WJ, Panoiu NC, Malloy KJ, Osgood RM, Brueck SRJ. Experimental demonstration of near-infrared negative-index metamaterials. Phys Rev Lett 2005;95:137404.

[3] Dolling G, Enkrich C, Wegener M, Soukoulis CM, Linden S. Low-loss negative-index metamaterial at telecommunication wavelengths. Opt Lett 2006;31:1800-2.

[4] Smith DR, Pendry JB, Wiltshire MCK. Metamaterials and negative refractive index. Science 2004;305:788-92.

[5] Shalaev VM. Optical negative-index metamaterials. Nat Photonics 2007;1:41-8. 
[6] Engheta N. Circuits with light at nanoscales: Optical nanocircuits inspired by metamaterials. Science 2007;317:1698-702

[7] Liu YM, Zhang X. Metamaterials: a new frontier of science and technology. Chem Soc Rev 2011;40:2494-507.

[8] Landy NI, Sajuyigbe S, Mock JJ, Smith DR, Padilla WJ. Perfect metamaterial absorber. Phys Rev Lett. 2008;100:207402.

[9] Liu N, Mesch M, Weiss T, Hentschel M, Giessen H. Infrared Perfect Absorber and Its Application As Plasmonic Sensor. Nano Lett 2010;10:2342-8.

[10] Liu XL, Starr T, Starr AF, Padilla WJ. Infrared Spatial and Frequency Selective Metamaterial with Near-Unity Absorbance. Phys Rev Lett 2010;104(20).

[11] Watts CM, Liu XL, Padilla WJ. Metamaterial Electromagnetic Wave Absorbers. Adv Mater 2012;24:OP98-120.

[12] Wu CH, Neuner B, Shvets G, John J, Milder A, Zollars B, et al. Large-area wide-angle spectrally selective plasmonic absorber. Phys Rev B 2011;84:075102.

[13] Zhao B, Wang LP, Shuai Y, Zhang ZM. Thermophotovoltaic emitters based on a twodimensional grating/thin-film nanostructure. Int J Heat Mass Tran 2013;67:637-45.

[14] Narayanaswamy A, Chen G. Surface modes for near field thermophotovoltaics. Appl Phys Lett 2003;82:3544-6.

[15] Liu XL, Tyler T, Starr T, Starr AF, Jokerst NM, Padilla WJ. Taming the Blackbody with Infrared Metamaterials as Selective Thermal Emitters. Phys Rev Lett 2011;107:045901.

[16] Polder D, Vanhove M. Theory of Radiative Heat Transfer between Closely Spaced Bodies. Phys Rev B. 1971;4(10):3303-14.

[17] Mulet JP, Joulain K, Carminati R, Greffet JJ. Enhanced radiative heat transfer at nanometric distances. Microscale Therm Eng 2002;6:209-22.

[18] Shen S, Narayanaswamy A, Chen G. Surface Phonon Polaritons Mediated Energy Transfer between Nanoscale Gaps. Nano Lett 2009;9:2909-13.

[19] Rousseau E, Siria A, Jourdan G, Volz S, Comin F, Chevrier J, et al. Radiative heat transfer at the nanoscale. Nat Photonics 2009;3:514-7.

[20] Fu CJ, Zhang ZM. Nanoscale radiation heat transfer for silicon at different doping levels. Int J Heat Mass Tran 2006;49:1703-18.

[21] Basu S, Lee BJ, Zhang ZM. Infrared Radiative Properties of Heavily Doped Silicon at Room Temperature. J Heat Trans-T ASME 2010;132:023301. 
[22] van Zwol PJ, Thiele S, Berger C, de Heer WA, Chevrier J. Nanoscale Radiative Heat Flow due to Surface Plasmons in Graphene and Doped Silicon. Phys Rev Lett 2012;109:264301.

[23] Svetovoy VB, van Zwol PJ, Chevrier J. Plasmon enhanced near-field radiative heat transfer for graphene covered dielectrics. Phys Rev B 2012;85:155418.

[24] Rytov, SM, Krastov, YA, Tatarskii, VI. Principles of Statistical Radiophysics. Berlin: Springer-Verlag; 1989.

[25] Joulain K, Drevillon J, Ben-Abdallah P. Noncontact heat transfer between two metamaterials. Phys Rev B 2010;81:165119.

[26] Pendry JB, Holden AJ, Stewart WJ, Youngs I. Extremely low frequency plasmons in metallic mesostructures. Phys Rev Lett 1996;76:4773-6.

[27] Pendry JB, Holden AJ, Robbins DJ, Stewart WJ. Magnetism from conductors and enhanced nonlinear phenomena. IEEE T Microw Theory 1999;47:2075-84.

[28] Francoeur M, Basu S, Petersen SJ. Electric and magnetic surface polariton mediated near-field radiative heat transfer between metamaterials made of silicon carbide particles. Opt Express. 2011;19:18774-88.

[29] Biehs SA, Ben-Abdallah P, Rosa FSS, Joulain K, Greffet JJ. Nanoscale heat flux between nanoporous materials. Opt Express 2011;19:A1088-A103.

[30] Liu XL, Bright TJ, Zhang ZM. Application Conditions of Effective Medium Theory in Near-Field Radiative Heat Transfer Between Multilayered Metamaterials. J Heat Trans-T ASME. 2014;136:092703.

[31] Smith DR, Schultz S, Markos P, Soukoulis CM. Determination of effective permittivity and permeability of metamaterials from reflection and transmission coefficients. Phys Rev B 2002;65:195104.

[32] Hao JM, Zhou L, Qiu M. Nearly total absorption of light and heat generation by plasmonic metamaterials. Phys Rev B. 2011;83:165107.

[33] Lambrecht A, Marachevsky VN. Casimir Interaction of Dielectric Gratings. Phys Rev Lett 2008;101:160403.

[34] Guerout R, Lussange J, Rosa FSS, Hugonin JP, Dalvit DAR, Greffet JJ, et al. Enhanced radiative heat transfer between nanostructured gold plates. Phys Rev B 2012;85:180301.

[35] Lussange J, Guerout R, Rosa FSS, Greffet JJ, Lambrecht A, Reynaud S. Radiative heat transfer between two dielectric nanogratings in the scattering approach. Phys Rev B 2012;86:085432. 
[36] Wen SB. Direct Numerical Simulation of Near Field Thermal Radiation Based on Wiener Chaos Expansion of Thermal Fluctuating Current. J Heat Trans-T ASME 2010;132:072704.

[37] Liu BA, Shen S. Broadband near-field radiative thermal emitter/absorber based on hyperbolic metamaterials: Direct numerical simulation by the Wiener chaos expansion method. Phys Rev B 2013;87:115403.

[38] Rodriguez AW, Ilic O, Bermel P, Celanovic I, Joannopoulos JD, Soljačić M, Johnson SG. Frequency-Selective Near-Field Radiative Heat Transfer between Photonic Crystal Slabs: A Computational Approach for Arbitrary Geometries and Materials. Phys Rev Lett 2011;107:114302.

[39] Didari A, Mengüç MP. Analysis of near-field radiation transfer within nano-gaps using FDTD method. J Quant Spectrosc Radiat Transf 2014;146:214-6.

[40] McCauley AP, Reid MTH, Krüger M, Johnson SG. Modeling near-field radiative heat transfer from sharp objects using a general three-dimensional numerical scattering technique. Phys Rev B 2012;85:165104.

[41] Edalatpour S, Čuma M, Trueax T, Backman R, Francoeur M. Convergence analysis of the thermal discrete dipole approximation. Phys Rev E 2015;91:063307.

[42] Chapuis PO, Volz S, Henkel C, Joulain K, Greffet JJ. Effects of spatial dispersion in near-field radiative heat transfer between two parallel metallic surfaces. Phys Rev B 2008;77:035431.

[43] Ye YQ, Jin Y, He SL. Omnidirectional, polarization-insensitive and broadband thin absorber in the terahertz regime. J Opt Soc Am B 2010;27:498-504.

[44] Hao JM, Wang J, Liu XL, Padilla WJ, Zhou L, Qiu M. High performance optical absorber based on a plasmonic metamaterial. Appl Phys Lett 2010;96:251104.

[45] Hu CG, Zhao ZY, Chen XN, Luo XG. Realizing near-perfect absorption at visible frequencies. Opt Express 2009;17:11039-44.

[46] Bai Y, Jiang YY, Liu LH. Multi-band near-field radiative heat transfer between two anisotropic fishnet metamaterials. J Quant Spectrosc Radiat Transf 2015;158:36-42.

[47] Zhou N, Kinzel EC, Xu X. Complementary bowtie aperture for localizing and enhancing optical magnetic field. Opt Lett 2011;36:2764-6.

[48] Johnson PB, Christy RW. Optical constants of the noble metals. Phys Rev B 1972;9:5056-70. 
[49] Ordal MA, Long LL, Bell RJ, Bell SE, Bell RR, Alexander RW, et al. Optical Properties of the Metals $\mathrm{Al}, \mathrm{Co}, \mathrm{Cu}, \mathrm{Au}, \mathrm{Fe}, \mathrm{Pb}, \mathrm{Ni}, \mathrm{Pd}, \mathrm{Pt}, \mathrm{Ag}, \mathrm{Ti}$, and $\mathrm{W}$ in the Infrared and Far Infrared. Appl Optics 1983;22:1099-119.

[50] Palik, ED. Handbook of Optical Constants of Solid. San Diego: Academic; 1998.

[51] Sakurai A, Zhao B, Zhang ZM. Resonant frequency and bandwidth of metamaterial emitters and absorbers predicted by an RLC circuit model. J Quant Spectrosc Radiat Transf 2014;149:33-40. 